\title{
New HErschel Multi-wavelength Extragalactic Survey of Edge-on Spirals (NHEMESES)
}

\author{
B. W. Holwerda ${ }^{1}$, S. Bianchi ${ }^{2}$, M. Baes ${ }^{3}$, R. S. de Jong ${ }^{4}$, \\ J. J. Dalcanton ${ }^{5}$, D. Radburn-Smith ${ }^{5}$, K. Gordon ${ }^{6}$ and M. Xilouris \\ ${ }^{1}$ European Space Agency (ESTEC), Keplerlaan 1, 2200 AV Noordwijk, The Netherlands \\ ${ }^{2}$ INAF-Arcetri Astrophysical Observatory, Florence, ${ }^{3}$ University of Gent, ${ }^{4}$ Astronomisch \\ Insitüt Potsdam, ${ }^{5}$ University of Washington, ${ }^{6}$ Space Telescope Science Institute, ${ }^{7}$ National \\ Observatory of Athens
}

email: benne.holwerda@esa.int

\begin{abstract}
Edge-on spiral galaxies offer a unique perspective on the vertical structure of spiral disks, both stars and the iconic dark dustlanes. The thickness of these dustlanes can now be resolved for the first time with Herschel in far-infrared and sub-mm emission. We present NHEMESES, an ongoing project that targets 12 edge-on spiral galaxies with the PACS and SPIRE instruments on Herschel. These vertically resolved observations of edge-on spirals will impact on several current topics.

First and foremost, these Herschel observations will settle whether or not there is a phase change in the vertical structure of the ISM with disk mass. Previously, a dramatic change in dustlane morphology was observed as in massive disks the dust collapses into a thin lane. If this is the case, the vertical balance between turbulence and gravity dictates the ISM structure and consequently star-formation and related phenomena (spiral arms, bars etc.). We specifically target lower mass nearby edge-ons to complement existing Herschel observations of high-mass edge-on spirals (the HEROES project).

Secondly, the combined data-set, together with existing Spitzer observations, will drive a new generation of spiral disk Spectral Energy Distribution models. These model how dust reprocesses starlight to thermal emission but the dust geometry remains the critical unknown.

And thirdly, the observations will provide an accurate and unbiased census of the cold dusty structures occasionally seen extending out of the plane of the disk, when backlit by the stellar disk. To illustrate the NHEMESES project, we present early results on NGC 4244 and NGC 891, two well studies examples of a low and high-mass edge-on spiral.
\end{abstract}

Keywords. (ISM:) dust, extinction ISM: structure galaxies: fundamental parameters (typical scales, dust mass) galaxies: individual (NGC 4244, NGC 891) galaxies: ISM galaxies: spiral galaxies: structure infrared: ISM submillimeter

Edge-on spiral galaxies offer a unique perspective on spiral disks. An observer can explore the vertical structure of both stars and ISM using different wavelengths. Dust is mechanically linked to the cold ISM (Allen et al. 1986; Weingartner \& Draine 2001). Dalcanton et al. (2004) used the appearance of dust lanes as a probe of vertical stability of spiral disks. They found that in massive spiral disks, the ISM collapses into a thin dustlane, while the less massive disks show more flocculant dust morphology. In addition, observations of edge-ons often reveal dark structures extending out of the plane of massive disks (e.g., Howk 1999), but the quantity of extra-planar dust has been impossible to constrain from extinction measures. 


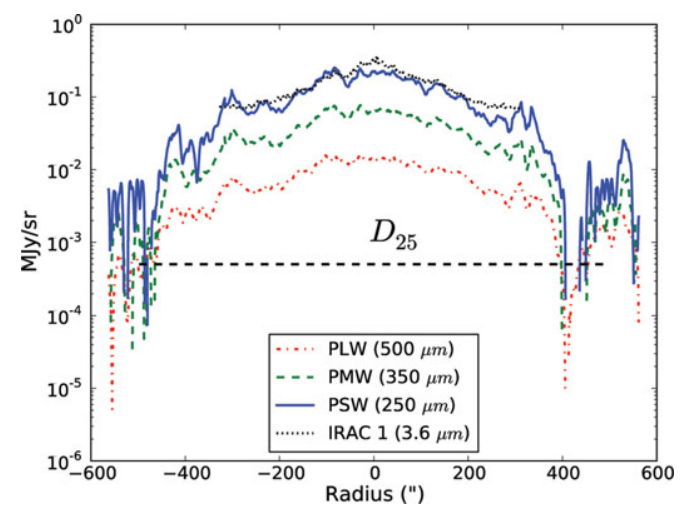

Figure 1. The radial profiles of NGC4244; Spitzer 3.6 (dotted line with closely spaced dots), SPIRE 250 (dotted), 350 (dashed) and 500 (solid) $\mu \mathrm{m}$.

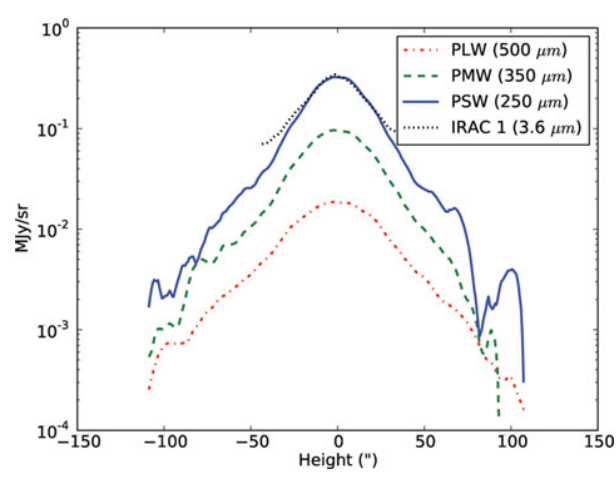

Figure 2. The vertical profile of NGC4244, same legend as Fig. 1. The SPIRE profile stays within the $3.6 \mu \mathrm{m}$ height.

A comprehensive approach is to model multi-wavelength images of edge-on spirals with a bulge, a stellar and a dust disk to constrain stellar and ISM structure (e.g., Xilouris et al. 1999; Bianchi 2008; Baes et al. 2010; Popescu et al. 2011). However, until now, these models are often degenerate in the vertical distributions of stellar light and ISM due to lack of resolution and wavelength coverage. With the advent of Herschel, the vertical structure of some nearby edge-on disks can be now resolved. Our Herschel program NHEMESES $†$ (PI B.W. Holwerda) aims to (1) measure the vertical scale of dust in spiral disks (an unknown in the above models), (2) the extra-planar dust component, and (3) whether or not the strong break seen in dust lanes by Dalcanton et al. (2004) is indeed a real phase-change in the cold ISM with disk mass.

We show some first NHEMESES results with the SPIRE morphology of NGC 4244, the prototypical disk-dominated low-mass spiral galaxy $\left(\mathrm{v}_{\mathrm{rot}}=95 \mathrm{kms}^{-1}\right)$.

The radial profile (Fig. 1) appears to truncate beyond a peak in flux from a small starformation region on both sides of the disks (see also Fig. 3). The radius of truncation is similar to those initially found by van der Kruit \& Searle (1981) and more recently by de Jong et al. (2007) for different stellar populations.

The vertical sub-mm profiles (Fig. 2) are similar in width to the Spitzer $3.6 \mu \mathrm{m}$ emission, a good tracer of the stellar mass (e.g., Meidt et al., this volume). Comerón et al. (2011) find evidence for a thick and thin stellar disk in this galaxy and the dusty ISM appears associated with the inner (thin) disk. In contrast, a second, thicker vertical component in NGC 891 has been reported (Kamphuis et al. 2007, Seon et al., this volume).

Fig. 3 shows the colour image based on the 250, 350 and $500 \mu \mathrm{m}$ SPIRE images with the HI contours from Zschaechner et al. (2011); Heald et al. (2011). The dust emission is restricted to the highest $\mathrm{HI}$ contour; it is a single, concentrated disk. This is in contrast to the more massive NGC 891, where Popescu \& Tuffs (2003); Bianchi \& Xilouris (2011) find evidence for dust throughout the HI disk and extended envelope (Oosterloo et al. 2007).

The fit to the Spectral Energy Distribution (SED) using the model of MacLachlan et al. (2011) (Fig. 4) finds an optically thin disk with a similar scale-height for both dust and stars (as did Seth et al. 2005, based on stellar populations). MacLachlan et al. report

$\dagger$ New HErshel Multi-wavelength Extragalactic Survey of Edge-on Spirals, a 10.3 hour, OT1, priority 2 program to supplement the GT program on large disks, HERshel Observations Edge-on Spirals, PI M. Baes. 


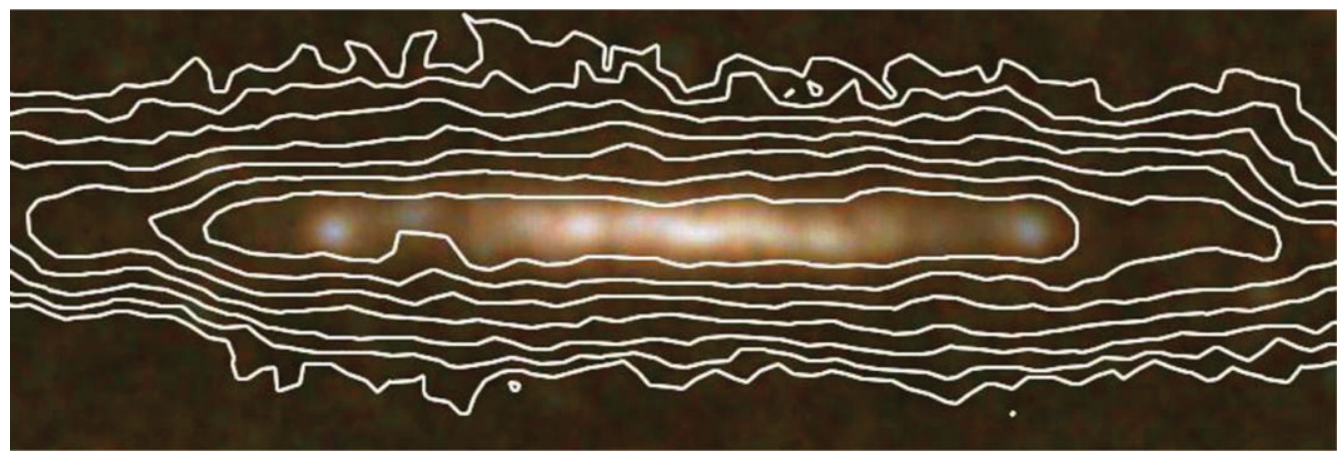

Figure 3. A three-colour image of the SPIRE observations of NGC 4244 with 250,350 and $500 \mu \mathrm{m}$ and the HI contours from Zschaechner et al. (2011) (contours at $6.4 \times 10^{19} \mathrm{~cm}^{-2}$ increasing by factors of 2). The SPIRE flux is contained mostly within the $8.1 \times 10^{19} \mathrm{~cm}^{-2}$ contour.

a dust mass of $2.38 \times 10^{6} M_{\odot}$ and a dust scale-length 1.8 times its stellar scale length. We find that their predicted sub-mm fluxes are lower than what we observe, possibly because the dusty ISM is much clumpier than the smooth diffuse ISM they assume for the SED model. However, we find that their high value of the scale-length of the dusty disk is comparable with the radial profile of sub-mm emission we observe (Fig. 1).

We find that dust in NGC 4244 is distributed vertically throughout the (thin) stellar disk, in accordance with the prediction from Dalcanton et al. (2004), but that, unlike the much more massive NGC 891, there is little evidence for a second dust component associated with the outer HI envelope. SED models for NGC 4244 are converging to a solution in which the large-scale distribution (in height and length) for the dust is comparable to the stellar ones, but distributed almost exclusively in clumps. This different distribution of the dusty geometry for lower-mass spiral disks may help explain the colder dust temperature and higher relative dust mass observed by H-ATLAS (Dunne et al. 2011, Dunne et al. this volume).

The NHEMESES project aims to observe 12 low-mass spirals with PACS and SPIRE on board Herschel. Combined with existing Herschel observations of massive edge-on

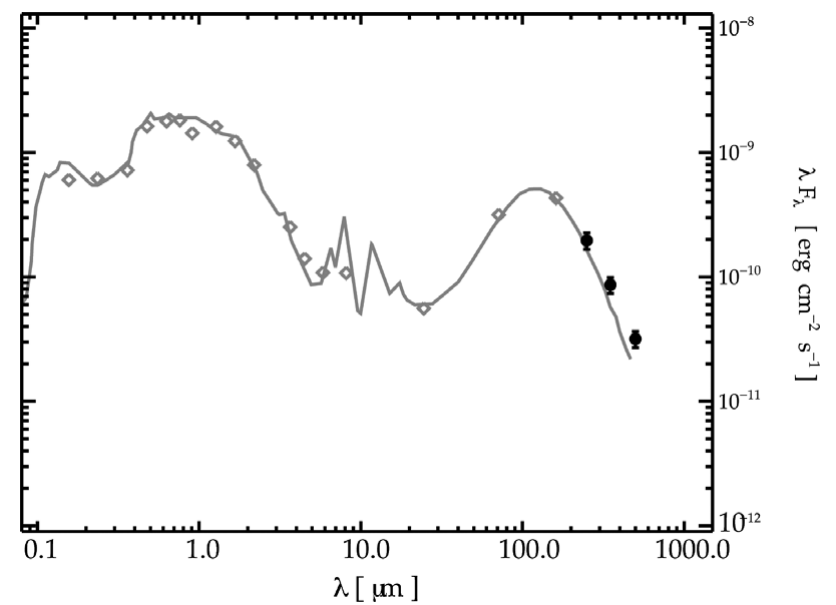

Figure 4. The Spectral Energy Distribution model from MacLachlan et al. (2011) (see also MacLachlan et al. (this volume) and the SPIRE fluxes for NGC 4244. The SED under-predicts the sub-mm fluxes by only a factor 2 . 
spiral galaxies, we aim to (1) ascertain if the ISM indeed goes through a phase-change at $120 \mathrm{~km} / \mathrm{s}$, (2) take a census of dusty outflows in nearby, quiescent, spiral disks and (3) provide a suite of multi-wavelength data (in combination with SDSS and Spitzer observations) to serve as a benchmark for SED models of edge-on spirals galaxies.

\section{References}

Allen, R. J. et al. 1986, Nature, 319, 296

Baes, M. et al. 2010, A\&A 518, L39+

Bianchi, S. 2008, A\&SA, 490, 461

Bianchi, S. \& Xilouris, E. M. 2011, A\&SA, 531, L11+

Comerón, S. et al. 2011, apj, 729, 18

Dalcanton, J. J. et al. 2004, ApJ, 608, 189

de Jong, R. S. et al. 2007, ApJ, 667, L49

Dunne, L. et al. 2011, MNRAS, 1395

Heald, G. et al. 2011, ArXiv e-prints

Howk, J. C. 1999, ApधSSS, 269, 293

Kamphuis, P. et al. 2007, A\&SA, 471, L1

MacLachlan, J. M. et al. 2011, ArXiv e-prints

Oosterloo, T. et al. 2007, AJ, 134, 1019

Popescu, C. C. \& Tuffs, R. J. 2003, A\&A, 410, L21

Popescu, C. C. et al. 2011, A\&BA, 527, A109+

Seth, A. C. et al. 2005, AJ, 129, 1331

van der Kruit, P. C. \& Searle, L. 1981, A\&A, 95, 105

Weingartner, J. C. \& Draine, B. T. 2001, ApJ, 553, 581

Xilouris, E. M. et al. 1999, A\& $A, 344,868$

Zschaechner, L. K. et al. 2011, ArXiv e-prints 